\title{
Modification of the mechanical properties of zirconium dioxide ceramics by means of multiwalled carbon nanotubes
}

\author{
E. A. Lyapunova ${ }^{1,2}$, S. V. Uvarov ${ }^{1}$, M. V. Grigoriev ${ }^{3,4}$, S. N. Kulkov ${ }^{3}$, O. B. Naimark ${ }^{1}$ \\ ${ }^{1}$ Institute of Continuous Media Mechanics, Ural Branch of Russian \\ Academy of Sciences, Perm, Russia \\ ${ }^{2}$ Ural Federal University, Ekaterinburg, Russia \\ ${ }^{3}$ Institute of Strength Physics and Materials Science, Siberian Branch of Russian \\ Academy of Sciences, Tomsk, Russia \\ ${ }^{4}$ Tomsk Polytechnic University, Tomsk, Russia \\ lyapunova@icmm.ru
}

PACS 62.23.Pq, 62.20.Qp, 63.22.Gh

DOI 10.17586/2220-8054-2016-7-1-198-203

\begin{abstract}
A ceramic matrix composite based on zirconium dioxide doped with carbon nanotubes (CNTs) and metallic silver as a plastic binder was produced by hydrothermal synthesis from ceramic precursor and a CNTs suspension followed by critical point drying of the synthesized hydrogel and metallic silver deposition on ceramic composite aerogel fragments from a $\mathrm{AgNO}_{3}$ solution. Multi-indentation loading of the composite has revealed two types of mechanical response: 1) hardness decreasing with an increasing of number of cycles and 2) significant increasing of hardness with an increasing number of indentations. Local chemical composition analysis has revealed correlations between the composite hardness and the presence oxygen atoms for first type and silver and yttrium atoms for second type of mechanical response respectively.
\end{abstract}

Keywords: carbon nanotubes, zirconium dioxide, hydrothermal synthesis, aerogel, nanoindentation.

Received: 20 November 2015

\section{Introduction}

The production of ceramic composites reinforced by carbon nanotubes (CNTs) is one of rapidly developing area in modern material science because of their ability to enhance ceramic structure, intensify sintering processes and decrease crystallite size [1-3]. Although addition of CNTs can change electromagnetic, electro- and heat-conductive properties of convenient ceramics, the current work is focused on mechanical properties of CNTs-modified zirconia composite. There is discrepancy in the literature concerning the ability of CNTs to improve the mechanical properties of such a composite [4,5], which apparently occurs because complete dispersion of CNTs agglomerates and their optimal arrangement in ceramic matrix can hardly be achieved. That is why reports about several orders of magnitude improvement of mechanical properties (fracture toughness, hardness, ets.) for CNTs-doped ceramics in comparison with conventional ceramics, as well as reports showing the absence of any significant effect of CNTs, can be found in literature. The aim of the present work is the development of the optimal mutual configuration of CNTs and ceramic crystallites in ceramic/CNTs composite and estimation of its mechanical behavior under multiple indentation loading. 


\section{Composite preparation}

Preparation of the composite includes the following steps: hydrothermal synthesis of dense hydrogel from suspension of zirconium salt and CNTs $(0.2 \mathrm{wt} \%$ concentration of CNTs); critical point drying of hydrogel and thermal treatment of obtained $\mathrm{ZrO}_{2} / \mathrm{CNTs}$ aerogel (these steps are described in detail in [6]); addition of metallic binder between aerogel fragments; and final consolidation of them into bulk samples by hot pressing. Critical point drying provided ceramic crystallites size less than $20 \mathrm{~nm}$ (Fig. 1, left) and preserves CNTs mutual configuration formed during hydrothermal synthesis.
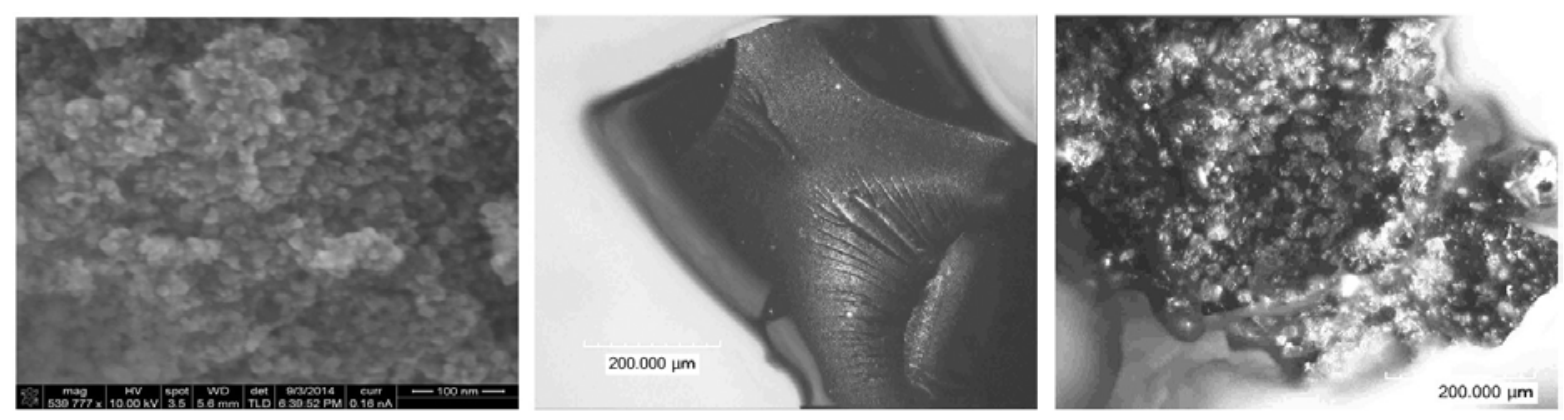

Fig. 1. Crystallite structure of synthesized aerogel (left), aerogel fragments before (center) and after (right) silver deposition

Metallic binder was added to the ceramic composite in order to increase material toughness. Such effect of small amount of titanium in zirconia ceramics was reported more than 50 years ago [7], but at that time, convenient ceramic powder with several hundred micron crystallites size and metallic powder with comparable grain size were used. In our case, with tiny size of ceramic crystallites, another method of metal particle addition was implemented; fragments of $\mathrm{ZrO}_{2} / \mathrm{CNTs}$ aerogels were immersed in $\mathrm{AgNO}_{3}$ solution and exposed to ultraviolet radiation with simultaneous sonication for process homogeneity. Such treatment leads to metallic silver precipitation on ceramic fragments (see: Fig. 1, center, Fig. 1, right); metal/ceramics ratio in final composite is controlled by ceramic powder size (its surface area), $\mathrm{AgNO}_{3}$ concentration and ultraviolet radiation intensity.

Consolidation of $\mathrm{ZrO}_{2} / \mathrm{CNTs}$ aerogel fragments after metal deposition was carried out by hot pressing in a graphite die at $1500{ }^{\circ} \mathrm{C}$ and $53.5 \mathrm{MPa}$ maximum pressing stress with 15 minutes dwelling time at the maximum temperature and pressure. X-ray spectroscopy of the sintered composite samples indirectly confirms the preservation of the ultrathin size of ceramic crystallites; there are peaks which correspond solely to the cubic phase of zirconium dioxide (Fig. 2). Since the sintering temperature was much higher than the melting point of silver, the latter becomes liquid, covering nearby ceramic composite fragments and fills the space between them (Fig. 3). There are different scales of porosity in the sintered composite: the lower scale (from ten to a few hundred nanometer size) which corresponds to the $\mathrm{ZrO}_{2} / \mathrm{CNTs}$ aerogel fragments' structure (Fig. 3, right) and is provided in the initial steps of composite production (hydrothermal synthesis and critical point drying of hydrogel). The higher scale of porosity (from a few to several tens of micrometers, Fig. 3, left) is due to incomplete consolidation of ceramic composite fragments during sintering as long as the applied pressure and temperature are not sufficient to reach complete densification of the material.

Transmission electron microscopy (TEM) analysis of composite fragments after hot pressing confirmed the presence of CNTs in the material (Fig. 4, left), however other nanocarbon objects were found, namely carbon nanotoroids (Fig. 4, right). One of the possible reasons 


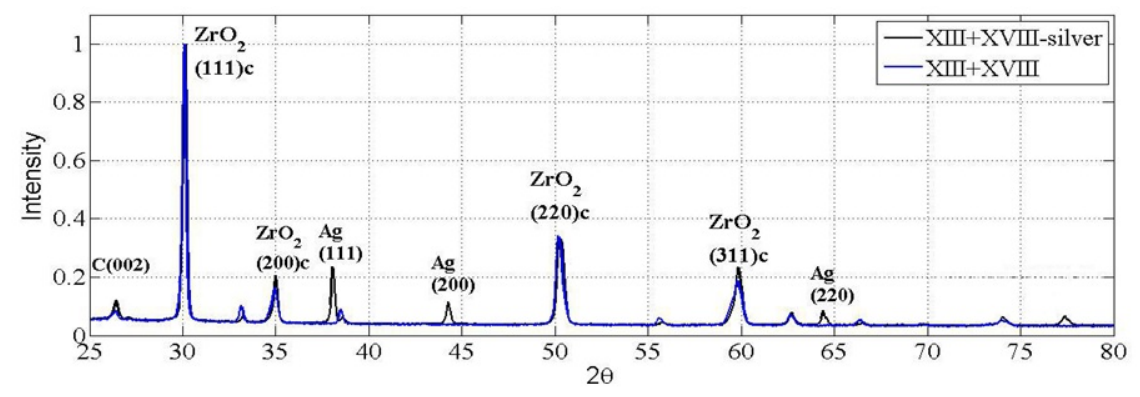

FIG. 2. X-ray spectra of $\mathrm{ZrO}_{2} / \mathrm{CNTs}$ composite (blue) and $\mathrm{ZrO}_{2} / \mathrm{CNTs}$ aerogel with metallic binder (black)
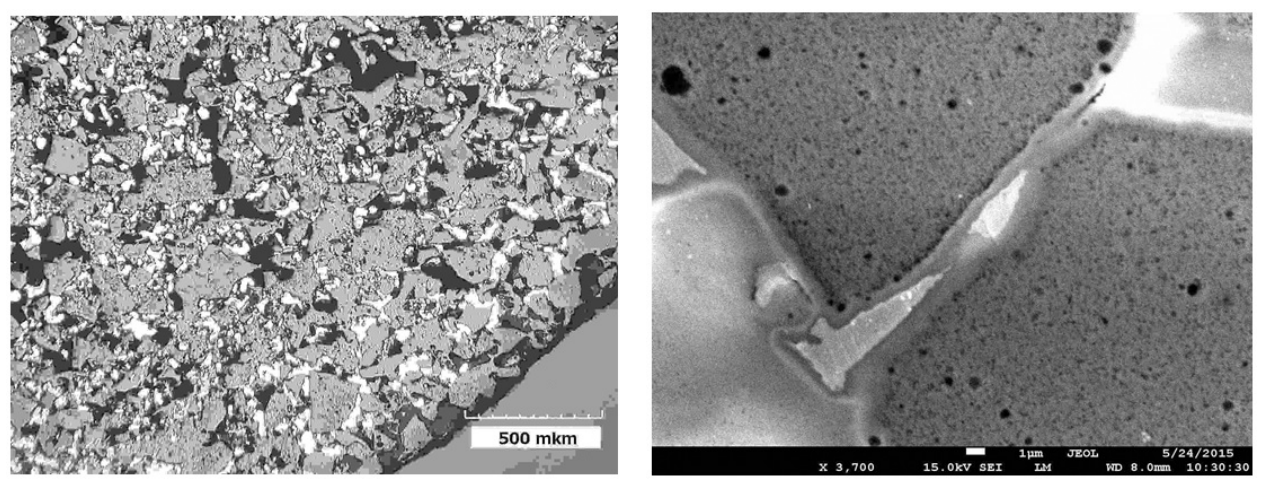

FIG. 3. Polished sample surface. Optical and scanning electron microscopy images demonstrating the common structure on the periphery of the sample (left) and the linkage between two ceramic aerogel fragments (right)

for their appearance is the high gradient magnetic field which occurred during heating of the graphite die by induction currents. CNTs toroidal and coiled structures are under keen investigation, since they are expected to have rather special electromagnetic properties [8].

The synthesis of $\mathrm{ZrO}_{2} / \mathrm{CNTs}$ aerogel and its preliminary structural characterization were conducted at the University of Louisville, USA. Metallic silver deposition on $\mathrm{ZrO}_{2} / \mathrm{CNTs}$ aerogel fragments was carried out at the Institute of continuous media mechanics, Perm, Russia. Hot pressing of aerogel fragments after silver deposition, $X$-ray spectroscopy and TEM of the sintered composite were performed at the Institute of Physical Foundations of Strength, Tomsk, Russia.

\section{Indentation experiment}

Mechanical experiments on multiple indentation of composite were realized on the basis of indentation system NanoTest (Perm State University, Perm, Russia) and consisted of the following steps: loading the sample with Berkovitch indenter to maximum force $F_{\max }$, dwelling the material at this load for 60 seconds, unloading the sample to $0.2 F_{\max }$, dwelling at this load for 60 seconds and repeating of the loading cycle again. Several series of experiments were produced varying the loading velocity $(2,4$ and $8 \mathrm{mN} / \mathrm{s})$ and number of cycles $(20$ and 40$)$ with the same maximum load $500 \mathrm{mN}$. For each loading conditions 30 experiments with $50 \mu \mathrm{m}$ istance were carried out. Fig. 5 shows the scanning electron microscope (SEM) image of the examined composite with a fragment of the indents map. Structural inhomogeneity provides some variation in measurement of mechanical properties, but comparison of force curves with 

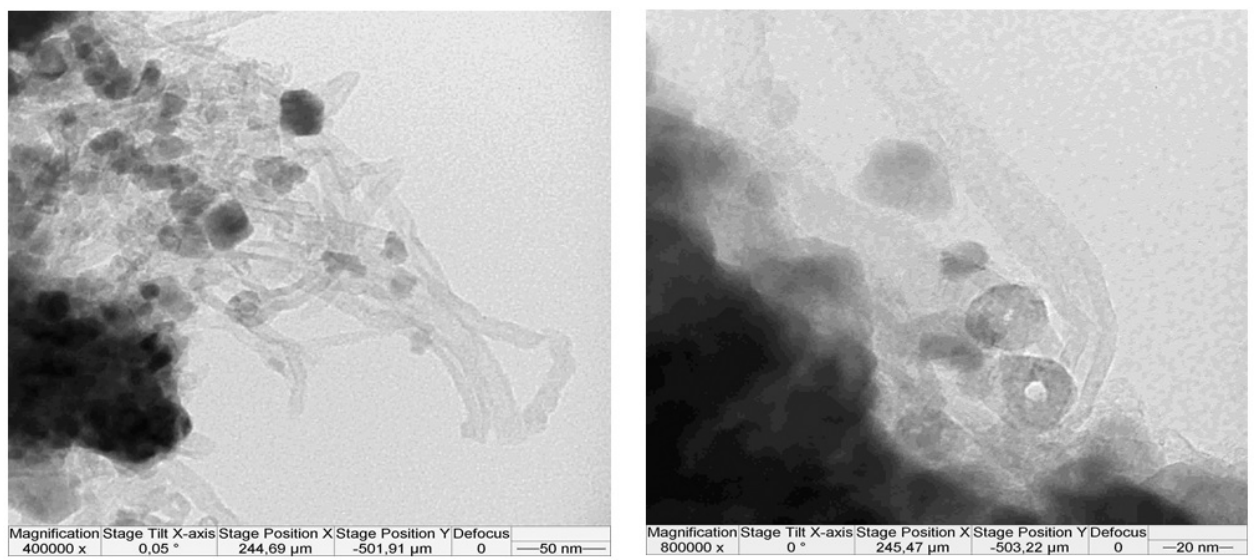

FIG. 4. CNTs with $\mathrm{ZrO}_{2}$ crystalites (left) and CNTs with carbon nanotoroids (right) in sintered material

SEM images of indents can help to eliminate unreliable points like indentation on the boundary between ceramic fragments and to analyze only the valuable points.

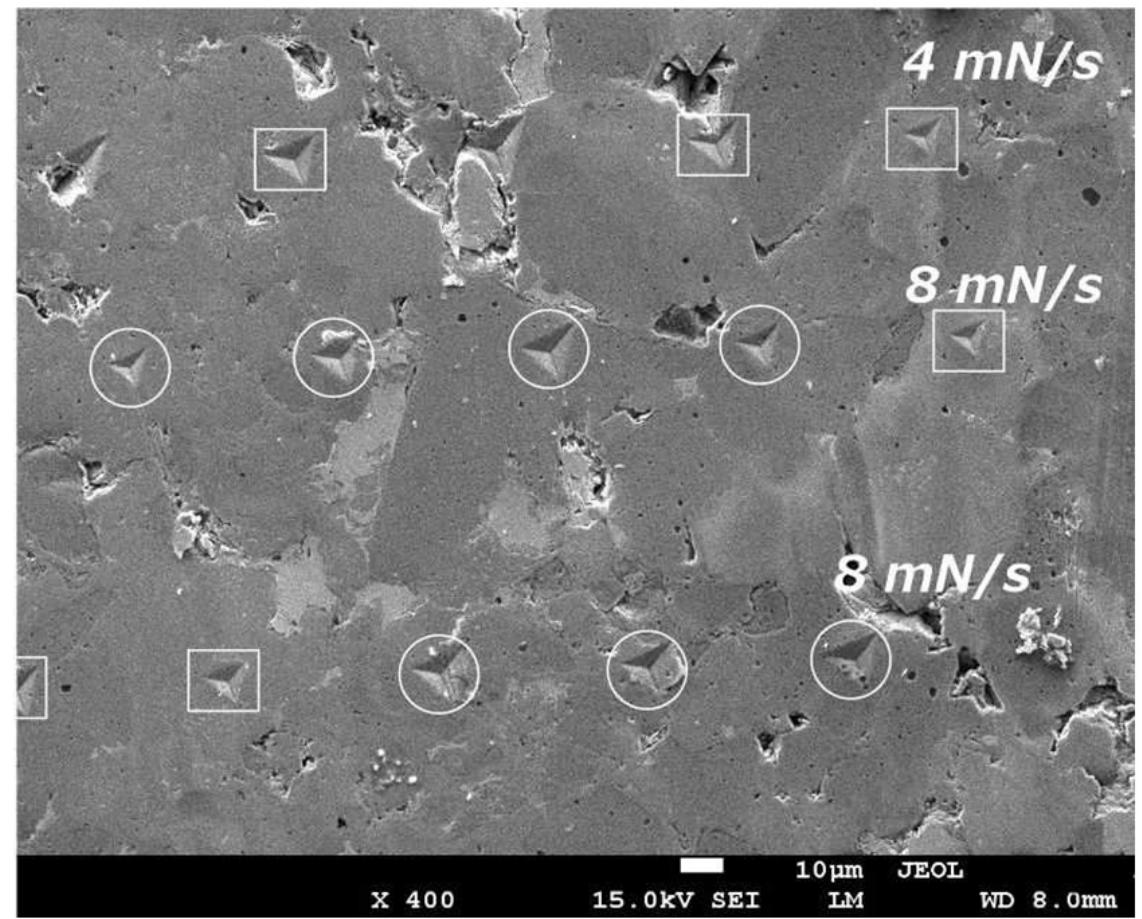

FIG. 5. Three series of indents on the composite produced under different loading conditions. Indents with first and second types of mechanical response are framed in squares and rounds respectively

The synthesized composite exhibits two types of mechanical response under multiple indentation: the first type has decreasing hardness (penetration depth increasing) with each indentation cycle (Fig. 6), and opposite one, namely increasing hardness (penetration depth decreasing) with increasing number of indentation cycles (Fig. 7). Analysis of local chemical composition near indents has shown correlations between the structural elements composition and mechanical properties of the material. Indeed, for the first type of mechanical response, there is a correlation of oxygen concentration with hardness values: the lower the oxygen 
concentration, the higher the hardness of material. For the second type of mechanical response, with anomalous composite hardening, the correlation of silver and yttrium concentrations with hardness values has been found, namely, the higher the silver and yttrium percentage, the higher the hardness of material.
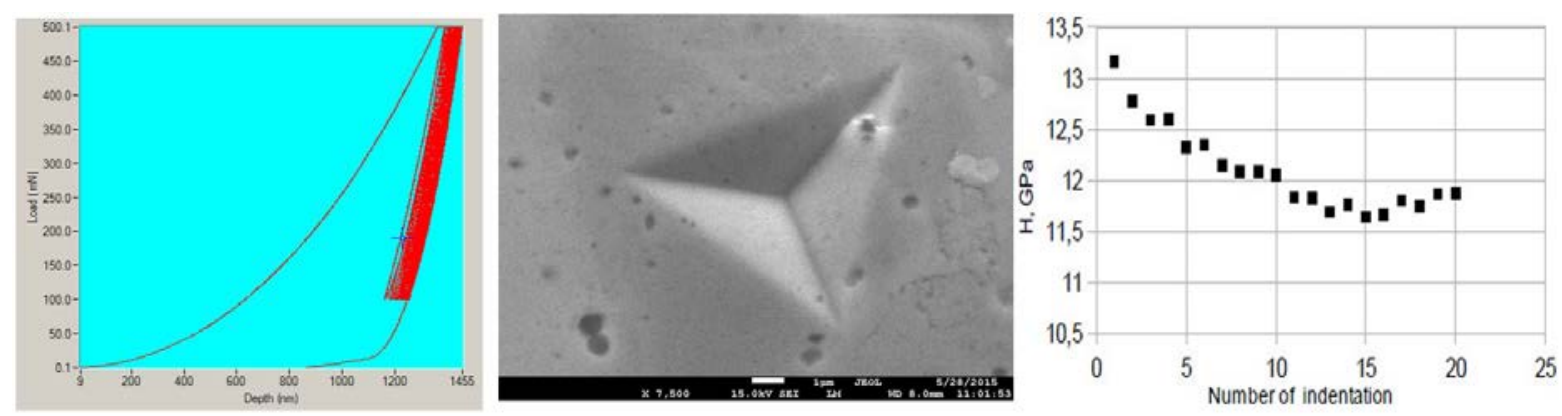

FIG. 6. Force curve (left), SEM image of indent (center) and evolution of hardness during the multiple indentation experiment (right) for the first type of material response
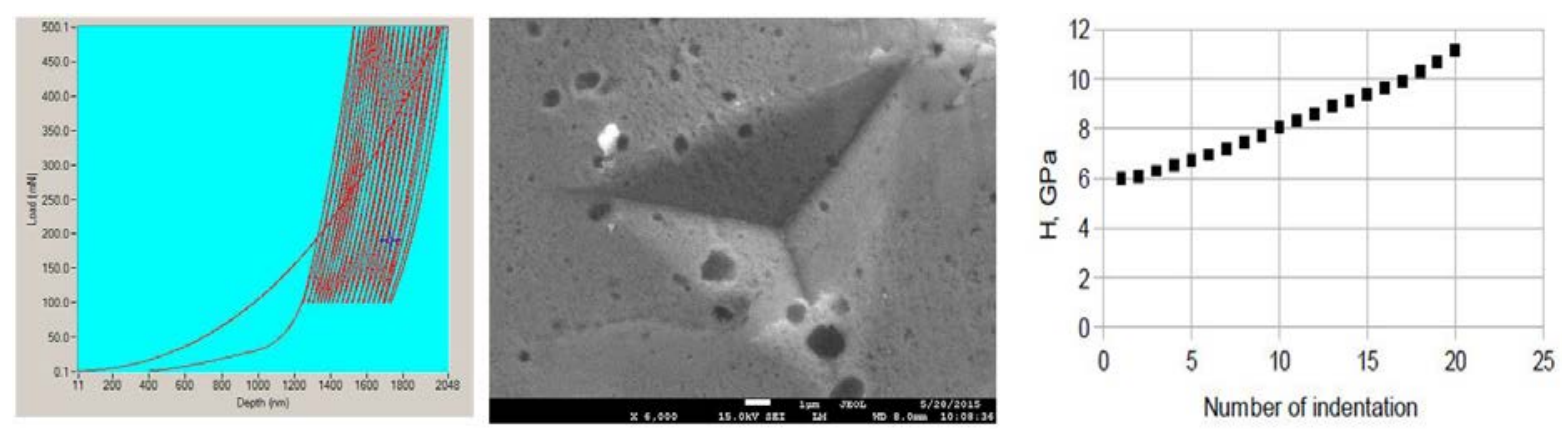

FIG. 7. Force curve (left), SEM image of indent (center) and evolution of hardness during the multiple indentation experiment (right) for the second type of material response

One of the possible explanation for this can be the following: the first type of mechanical response is governed mainly by oxygen vacancies in the ceramic matrix, while anomalous hardening is the result of the phase composition of ceramics composite (which is governed by yttrium concentration) and the presence of silver atoms. Analysis of mutual configuration of metallic particles and CNTs in ceramic matrix as well as investigation of additional chemical bonds forming between them can prove this hypothesis. Also, there is no increasing of hardness during multiple loading of conventional ceramics, so one can conclude that CNTs play a significant role in such type of mechanical response.

\section{Conclusions}

The ceramic matrix composite based on CNTs-doped zirconium dioxide and metallic silver as a plastic binder was produced. Ultrathin ceramic crystallites (size less than $20 \mathrm{~nm}$ ) were formed by means of hydrothermal synthesis and critical point drying of the ceramic composite precursor. Multi-indentation loading of composite has revealed two types of mechanical response: 1) hardness decreasing (penetration depth increasing) with each cycle of indentation, and 2) increasing of hardness (penetration depth decreasing) with increasing number of 
indentations. Such different mechanical behavior is attributed to both chemical and structural inhomogeneity of material. Local chemical composition analysis has revealed correlations between hardness changes and the presence of silver and yttrium or oxygen atoms for different types of mechanical responses.

\section{Acknowledgements}

This work is supported by Russian Foundation for Basic Research (grant N14-01-96015ural-a).

\section{References}

[1] Xia Z., Riester L., Curtin W.A., Li H., Sheldon B.W., Liang J., Chang B., Xu J.M. Direct observation of toughening mechanisms in carbon nanotube ceramic matrix composites. Acta Mater., 2004, 52(4), P. 931-944.

[2] Lyapunova E., Naimark O., Kulkov S., Dedova E., Sobolev I. Structure of zirconium oxide-multiwalled carbon nanotubes composite produced by electrophoretic deposition. Inorg. Mater, 2015, 51(1), P. $20-24$.

[3] Vasiliev A.L., Poyato R., Padture N.P. Single-wall carbon nanotubes at ceramic grain boundaries. Scripta Mater, 2007, 56, P. 461-463.

[4] Curtin W.A., Sheldon B.W. CNT-reinforced ceramics and metals. Materials Today, 2004, 7(11), P. 44-49.

[5] Peigney A., Laurent C., Flahaut E., Rousset A. Carbon nanotubes in novel ceramic matrix nanocomposites. Ceramic Intern., 2000, 26, P. 677-683.

[6] Lyapunova E., Lunegov I., Uvarov S., Naimark O. Structural aspects of zirconium dioxide/MWCNTs composite. Actual problems of condensed media physics, 2015, P. 83-90. ICMM UB of RAS, Perm (in Russian).

[7] Weber B.C., Garrett H.J., Mauer F.A., Schwartz M.A. Observations on the stabilization of zirconia. J. Amer. Ceramic Soc., 1956, 39(6), P. 197-206.

[8] Liu L., Liu F., Zhao J. Curved carbon nanotubes: from unique geometries to novel properties and peculiar applications. Nano Research, 2014, 7(5), P.626-657. 